\title{
Use of Intraoperative Tranexamic Acid and Wound Complications in Spine Surgery: A Retrospective Cohort Study
}

\author{
Moti Kramer ${ }^{1}$, Michael Drexler ${ }^{1}$, Amir Herman ${ }^{1}$, Tal Kalimian $^{1}$, Yuri Klassov ${ }^{2}$, Layalee Abu Nasser ${ }^{2}$ \\ ${ }^{1}$ Department of Orthopedics, Assuta Ashdod University Medical Center, Ashdod, Israel \\ ${ }^{2}$ Department of Orthopedics, Soroka University Medical Center, Beer Sheva, Israel
}

\section{Study Design: A retrospective cohort study.}

Purpose: This study aims to examine the effect of tranexamic acid (TXA) on postoperative wound healing in spine surgery.

Overview of Literature: TXA (Cyklokapron, Hexakapron) is a widely used anti-fibrinolytic drug that is shown to be effective in mitigating hemorrhage during and after surgery by competitively blocking plasminogen in fibrinolytic cascade. Plasminogen also plays a role in inflammatory and infectious diseases. The modulation of this role by TXA may influence the development of postoperative infectious complications.

Methods: We collected and reviewed the charts of 110 patients who underwent spine surgery at our academic center. We used multivariate regression analysis to assess the factors affecting surgical site infection (SSI).

Results: Of the 110 patients included in this study, 21 patients (19\%) were categorized as having postoperative wound complications, 16 patients $(14 \%)$ had deep or superficial wound infection, and five patients $(4 \%)$ had wound dehiscence. Patients with a higher surgical invasiveness index score, longer surgeries, and older patients were found to be at risk for wound complications. TXA was determined not to be a direct risk factor for wound healing complications and SSIs.

Conclusions: We found no risk of wound healing complications and SSI directly attributable to preoperative and intraoperative treatment with TXA in spine surgeries.

Keywords: Tranexamic acid; Spine surgery; Surgical invasiveness index; Plasminogen; Fibrinolysis; Surgical wound dehiscence; Surgical site infection

\section{Introduction}

Surgical site infections (SSIs) and hardware infections are serious complications of spine surgery. They are, by definition, hospital-acquired infections that lead to high rates of morbidity, unfavorable surgical outcomes, extended length of hospital stays, and increased costs. The reported incidence of SSI after spinal surgery is between $0.7 \%$ and $15 \%$ depending on the type of procedure and the studied population [1-5].

Tranexamic acid (TXA; Cyklokapron, Hexakapron) is an anti-fibrinolytic drug that mitigates hemorrhaging during and after surgery. TXA is a synthetic analog of the amino acid lysine and acts as an anti-fibrinolytic agent by

Received Jul 24, 2019; Revised Nov 5, 2019; Accepted Nov 13, 2019

Corresponding author: Tal Kalimian

Department of Orthopedics, Assuta Ashdod University Medical Center, Harefua Street 7, Ashdod, Israel 7747629

Tel: +972-52-7875577, Fax: +972-72-3399093, E-mail: talkal@assuta.co.il 
competitively blocking lysine binding sites on plasminogen molecules, thereby reducing blood loss and transfusion requirements in major surgeries. It has become quite popular for intraoperative use in recent years [6-11]. TXA has been prescribed both intraoperatively and perioperatively in various spine procedures, including large-scale scoliosis correction surgeries $[9,12]$.

Plasminogen is a key molecule of fibrinolysis cascade; it is converted to the active enzyme plasmin by plasminogen activators. However, plasminogen also plays a role in the inflammatory response and has a critical role in the host response to infection [13-16]. While the anti-fibrinolytic effects of TXA have been described and are being promoted in the setting of major surgeries, the clinical effects of its use on inflammation and infection are less clear. Modulation of this inflammatory response by TXA may contribute to the development of infectious complications following surgery. The coagulation cascade is intricately associated with inflammatory pathways, and the antiinflammatory effects of TXA are poorly understood. Wound healing was severely impaired in plasminogendeficient mice [15-18], and treatment with TXA led to a distinct aggravation of staphylococcal septic arthritis and sepsis in mice [13]. However, as mentioned above, a large block of clinical evidence shows no such increase in the postoperative surgical site or deep infection in major surgeries such as knee and hip arthroplasties, spinal surgery, and scoliosis and trauma emergent surgeries [9,19-21]. Its safety and minor effect on wound healing were also examined in severe military battle-induced cases in which TXA was given in the pre-hospital setting before wound debridement and antibiotic treatment. No increased risk of infection was observed when compared with patients who were not treated with TXA after classifying the extent and severity of the injury [22].

This study aims to examine whether TXA affects postoperative wound healing and SSI rates in patients undergoing spine surgery at Soroka University Medical Center and to examine other risk factors for SSI and wound healing complications in our institute.

\section{Materials and Methods}

\section{Patients}

We conducted a retrospective cohort study based on chart reviews. The charts of 110 consecutive patients who un- derwent spine surgery from April 2015 to May 2016, by three different surgeons, at one academic medical center, were collected. The inclusion criteria included all spine surgeries that took place in this hospital, including trauma and elective and minimally invasive surgeries with and without instrumentation. The exclusion criteria included any revision case conducted due to infection, any surgery indicated due to a different presurgical spinal infection (i.e., epidural abscess, vertebral osteomyelitis, sepsis), and pediatric patients. The collected data includes demographics such as age and sex, chronic diseases and other comorbidities, surgery duration, hemoglobin level during surgery, dose administration of TXA on postoperative day 1 if given, and SSI if it occurred and whether it was superficial or deep. We collected data regarding the infecting organism identified by blood or deep wound cultures; superficial and deep wound infection were defined according to the Centers for Disease Control and Prevention (CDC) criteria for SSI (Fig. 1) [23,24]. The primary endpoint was defined as any SSI, deep or superficial, by CDC criteria.

Superficial incisional SSI

Infection occurs within 30 days after the operation and infection involves only skin and subcutaneous tissue of the incision and at least one of the following:

1. Purulent drainage with or without laboratory confirmation, from the superficial incision

2. Organisms isolated from an aseptically obtained culture of fluid or tissue from the superficial incision

3. At least one of the following signs or symptoms of infection: pain or tenderness, localised swelling, redness, or heat and superficial incision is deliberately opened by surgeon, unless incision is culture-negative

4. Diagnosis of superficial incisional SSI made by a surgeon or attending physician

Deep incisional SSI

Infection occurs within 30 days after the operation if no implant is left in place or within one year if implant is in place and the infection appears to be related to the operation and infection involves deep soft tissue (e.g., fascia, muscle) of the incision and at least one of the following:

1. Purulent drainage from the deep incision but not from the organ/space component of the surgical site

2. A deep incision spontaneously dehisces or is deliberately opened by a surgeon when the patient has at least one of the following signs or symptoms: fever $\left(>38^{\circ} \mathrm{C}\right)$, localised pain or tenderness, unless incision is culture-negative

3. An abscess or other evidence of infection involving the deep incision is found on direct examination, during reoperation, or by histopathologic or radiologic examination

4. Diagnosis of deep incisional SSI made by a surgeon or attending physician

Fig. 1. Centers for Disease Control and Prevention definitions for SSI. SSI, surgical site infection. 
Surgery was initially conducted on all patients by one or more of three practicing spine surgeons. Patients were divided into two groups: those who received TXA preoperatively and intraoperatively and those who did not receive TXA during the surgery. None of the patients received TXA postoperatively. The infection rate in each group was noted. We calculated the invasiveness index of each procedure as described and validated by Mizra et al. [25] to compare the different procedures [26].

Infected patients were treated according to the invasiveness of their disease (deep or superficial), bacterial growth, secretion rate, and clinical assessment. The treatment options included parenteral antibiotics alone or surgical debridement and lavage, followed by parenteral antibiotics.

\section{Statistical analysis}

Data analysis was performed by an experienced biostatistician using IBM SPSS ver. 23.0 (IBM Corp., Armonk, NY, USA). Categorical variables are presented as frequency (percent). Comparisons between groups of categorical variables were performed using the chi-square test or Fisher's exact test. The latter was used when the expected count in any cell was less than 5 . Continuous variables are presented as mean \pm standard deviation. Comparisons between groups were performed using the WilcoxonMann-Whitney rank-sum test. The multivariate logistic regression model was fitted using infection as the dependent covariate. The initial model included age, length of surgery, TXA above $1 \mathrm{~g}$, number of comorbidities, spine invasiveness index, time of administration of the preventive antibiotics, and TXA if given before skin incision. The Akaike information criteria were used for model selection for both eliminating and adding covariates in the model, considering all second-level interactions. The final model selected is presented below. All presented $p$-values are two-sided; a $p$-value of less than $0.05(p<0.05)$ is considered statistically significant.

\section{Results}

A total of 110 patients were included in this study; 21 patients (19\%) were defined as having postoperative wound complications. Of those, 16 patients (14\%) were defined as having a deep infection and were treated surgically by debridement and lavage, followed by 6 weeks of parenteral antibiotics. In eight patients (50\%) from this group, local cultures taken during surgery were negative. The remaining five patients (4\%) were defined as having superficial wound infection and were treated empirically for 2 weeks with parenteral antibiotics, followed by 2 weeks of oral antibiotics.

When the wound complication rate based on different comorbidities was compared, there was no significant difference between the groups. This was also the case when examining the propensity for SSI in cases of patients with diabetes mellitus, human immunodeficiency virus, hepatitis, alcohol abuse, and obesity (Table 1). The only demographic variant showing a significant statistical difference was age. Older patients exhibited more wound complications than younger ones in both groups (Table 1).

Surgical data analysis showed a higher wound complication rate in procedures with a greater invasiveness index score and hence longer surgical time. We observed 17 wound complications out of 76 patients in the TXA group (22.4\%) versus only four patients out of 34 (11.8\%) in the group that did not receive TXA. However, this finding is not statistically significant ( $p=0.041$ ) (Table 2 ).

When comparing the dose relation, we found that patients who received more than $1 \mathrm{~g}$ of TXA during the procedure had higher wound complications compared with those who received a dose of less than $1 \mathrm{~g}$ or did not receive TXA at all (13/43 [30\%] versus 8/67 [12\%], respectively). This finding is statistically significant $(p=0.017$ ).

The multivariate analysis with logistic regression showed that mean surgery time in patients who were given a single dose of $1 \mathrm{~g}$ or no TXA at all was $2.97 \pm 1.45$ hours, while in patients who were given more than one dose of TXA, the mean surgery time was $4.58 \pm 1.83$ hours. This difference is also statistically significant $(p<0.001)$ and may explain why TXA above $1 \mathrm{~g}$ is associated with a higher SSI rate. The TXA dose was a confounder; therefore, the actual variable that influenced the wound complication incidence was surgery time. We found that it was not the TXA dose but rather the longer surgical time that increased the risk of infection (Table 3 ).

Blood transfusions and the urgency of the procedure were not associated with a higher wound complication rate. This might be attributed to the fact that only one patient received a postoperative blood transfusion and the average age of the patients who underwent an emergency procedure was lower than those undergoing elective procedures (Table 2). 
Table 1. Demographic and clinical data

\begin{tabular}{|c|c|c|c|c|}
\hline Characteristic & Total & No surgical site infection & Surgical site infection & $p$-value \\
\hline No. of patients & $110(100.0)$ & $89(80.9)$ & $21(19.1)$ & \\
\hline Age (yr) & $52.57 \pm 17.51$ & $50.84 \pm 17.21$ & $59.93 \pm 17.25$ & 0.039 \\
\hline \multicolumn{5}{|l|}{ Diabetes mellitus } \\
\hline No & $84(76.4)$ & $10(83.3)$ & $14(16.7)$ & \\
\hline Yes & $26(23.6)$ & $19(73.1)$ & $7(26.9)$ & 0.245 \\
\hline \multicolumn{5}{|l|}{ Hypothyroidism } \\
\hline No & $103(93.6)$ & $84(81.6)$ & $19(18.4)$ & \\
\hline Yes & $7(6.4)$ & $5(71.4)$ & $2(28.6)$ & 0.510 \\
\hline \multicolumn{5}{|l|}{ Dyslipidemia } \\
\hline No & $102(92.7)$ & $83(81.4)$ & $19(18.6)$ & \\
\hline Yes & $8(7.3)$ & $6(75.0)$ & $2(25.0)$ & 0.659 \\
\hline \multicolumn{5}{|c|}{ Chronic obstructive pulmonary disease/asthma } \\
\hline No & $103(93.6)$ & $83(80.6)$ & $20(19.4)$ & \\
\hline Yes & $7(6.4)$ & $6(6.7)$ & $1(14.3)$ & 0.738 \\
\hline \multicolumn{5}{|l|}{ Obesity } \\
\hline No & $104(94.5)$ & $84(80.8)$ & $20(19.2)$ & \\
\hline Yes & $6(5.5)$ & $5(5.6)$ & $1(4.8)$ & 0.877 \\
\hline \multicolumn{5}{|c|}{ Ischemic heart disease } \\
\hline No & $103(93.6)$ & $84(81.6)$ & $19(18.4)$ & \\
\hline Yes & $7(6.4)$ & $5(71.4)$ & $2(28.6)$ & 0.510 \\
\hline \multicolumn{5}{|l|}{ Arrhythmia } \\
\hline No & $104(94.5)$ & $85(81.7)$ & $19(18.3)$ & \\
\hline Yes & $6(5.5)$ & $4(66.7)$ & $2(33.3)$ & 0.361 \\
\hline \multicolumn{5}{|c|}{ Connective tissue disease } \\
\hline No & $104(94.5)$ & $83(79.8)$ & $21(20.2)$ & \\
\hline Yes & $6(5.5)$ & $6(100.0)$ & 0 & 0.221 \\
\hline \multicolumn{5}{|l|}{ Hypertension } \\
\hline No & $80(72.7)$ & $68(85.0)$ & $12(15.0)$ & \\
\hline Yes & $30(27.3)$ & $21(70.0)$ & $9(30.0)$ & 0.075 \\
\hline \multicolumn{5}{|l|}{ Renal failure } \\
\hline No & $107(97.3)$ & $86(80.4)$ & $21(19.6)$ & \\
\hline Yes & $3(2.7)$ & $3(100.0)$ & 0 & 0.394 \\
\hline \multicolumn{5}{|c|}{ Human immunodeficiency virus } \\
\hline No & $108(98.2)$ & $88(81.5)$ & $20(18.5)$ & \\
\hline Yes & $2(1.8)$ & $1(50.0)$ & $1(50.0)$ & 0.262 \\
\hline \multicolumn{5}{|l|}{ Hepatitis } \\
\hline No & $105(95.5)$ & $84(80.0)$ & $21(20.0)$ & \\
\hline Yes & $5(4.5)$ & $5(5.6)$ & 0 & 0.266 \\
\hline \multicolumn{5}{|l|}{ Alcohol abuse } \\
\hline No & 108 (98.2) & $87(80.6)$ & $21(19.4)$ & \\
\hline Yes & $2(1.8)$ & $2(2.2)$ & 0 & 0.488 \\
\hline \multicolumn{5}{|l|}{ No. of comorbidities } \\
\hline 0 & $58(52.7)$ & $50(86.2)$ & $8(13.8)$ & \\
\hline 1 & $16(14.5)$ & $13(81.3)$ & $3(18.8)$ & \\
\hline$\geq 2$ & $36(32.7)$ & $26(72.2)$ & $10(27.8)$ & 0.245 \\
\hline
\end{tabular}

Values are presented as number (\%) or mean \pm standard deviation. 
Table 2. Surgery related data

\begin{tabular}{|c|c|c|c|c|}
\hline Variable & Total & No surgical site infection & Surgical site infection & $p$-value \\
\hline Spine invasiveness index & $7.6 \pm 3.91$ & $7.13 \pm 3.81$ & $9.57 \pm 3.8$ & 0.007 \\
\hline Sugery time (hr) & $3.61 \pm 1.79$ & $3.33 \pm 1.68$ & $4.8 \pm 1.8$ & 0.001 \\
\hline TXA $(g)$ & $1.22 \pm 1.13$ & $1.14 \pm 1.15$ & $1.57 \pm 0.98$ & 0.041 \\
\hline Time from prophylactic antibiotic to cut (min) & $61.48 \pm 33.7$ & $60.6 \pm 33.1$ & $64.9 \pm 37.0$ & 0.721 \\
\hline Time from TXA to cut (min) & $20.9 \pm 53.5$ & $18.8 \pm 55.8$ & $27.7 \pm 45.9$ & 0.664 \\
\hline \multicolumn{5}{|l|}{ TXA } \\
\hline No given & $34(30.9)$ & $30(88.2)$ & $4(11.8)$ & \\
\hline Given & $76(69.1)$ & $59(77.6)$ & $17(22.4)$ & 0.191 \\
\hline \multicolumn{5}{|l|}{ TXA } \\
\hline$\leq 1$ & $67(60.9)$ & $59(88.1)$ & $8(11.9)$ & \\
\hline$>1$ & $43(39.1)$ & $30(69.8)$ & $13(30.2)$ & 0.017 \\
\hline \multicolumn{5}{|l|}{ Urgent surgery } \\
\hline No & $47(42.7)$ & $39(83.0)$ & $8(17.0)$ & \\
\hline Yes & $63(57.3)$ & $50(79.4)$ & $13(20.6)$ & 0.633 \\
\hline \multicolumn{5}{|l|}{ Blood transfusion } \\
\hline No & 109 (99.1) & $89(81.7)$ & $20(18.3)$ & \\
\hline Yes & $1(0.9)$ & 0 & $1(100.0)$ & 0.191 \\
\hline
\end{tabular}

Values are presented as mean \pm standard deviation or number $(\%)$.

TXA, tranexamic acid.

Table 3. Multivariate analysis logistic regression

\begin{tabular}{llcr} 
Variable & Beta & Odds ratio (95\% confidence interval) & $p$-value \\
Age $(\mathrm{yr})$ & -0.0616 & $0.940(0.874-1.011)$ & 0.1030 \\
Surgery time (hr) & 0.432 & $1.540(1.055-2.248)$ & 0.0249 \\
\hline Spine invasiveness index & -0.6352 & $0.529(0.298-0.940)$ & 0.0300 \\
Age spine invasiveness index & 0.01133 & $1.011(1.002-1.020)$ & 0.0172 \\
\hline
\end{tabular}

The mean surgery time in patients given TXA $\leq 1$ was $2.97 \pm 1.45$ hours, while in patients who received TXA $>1$ the mean surgery time was $4.58 \pm 1.83$ hours. This difference was statistically significant $(p$-value $<0.001)$. This explains why TXA above $1 \mathrm{~g}$ was associated with more infections while it was not included in the multivariate model. TXA dosage above $1 \mathrm{~g}$ was a confounder while the actual variable which influences infection is surgery time.

TXA, tranexamic acid

Surgeries with a higher surgical invasiveness index score and surgeries performed on older patients had more wound complications. This difference is also significant after performing the regression analysis (Table 3).

The time of antibiotic administration before skin incision and TXA administration before and after skin incising showed no statistical significance between the groups $(64$ minutes in the wound complication group versus $60 \mathrm{~min}$ utes in the no complication group; $p=0.07$ ), and all were within the range of the recommended preoperative time [5].

\section{Discussion}

Wound complications and SSI are serious complications after spine surgery. These are hospital-acquired infections that may cause severe morbidity and poor quality of life, mortality, extended length of hospitalization, and increased costs.

This study reinforces data describing comorbidities such as patient age and surgery time and complexity (as expressed by the invasiveness index), which is known to be associated with increased wound complications. This study aims to examine the effect of preoperative TXA on 
wound complication rate. The SSI rates were $22.4 \%$ and $11.8 \%$ for patients who were treated with or without TXA, respectively. Although the results show a tendency for infection in patients treated with TXA, this distinction in the infection rate did not reach statistical significance $(p=0.191)$. This may be due to the small sample size, and further studies are encouraged. According to our results, we conclude that preoperative TXA use does not result in statistically significant increased wound complications during and after spine surgery. Although our results showed a positive statistically significant dose relationship between TXA and wound complication, this is probably related to other comorbidities and is, therefore, not a factor in and of itself. It should be emphasized that in surgical procedures of longer duration and a higher invasiveness index score, patients usually received more than one dose of TXA, which is a probable explanation for this finding. The increased rate of SSI in patients who received higher doses of TXA compared with those who received lower doses is merely a reflection of the longer surgical time, which has been proven to be a risk factor for SSI [27-29].

The incidence of SSI following spinal surgery has been reported to be between $0.7 \%$ and $12 \%$ depending on the type of procedure, the studied population, and various risk factors reported [1-4]. These risk factors include the sex of the patient (female), inpatient status, patients with insulin-dependent diabetes, preoperative steroid use for more than 10 days, hematocrit $<35 \%$, body mass index $>30 \mathrm{~kg} / \mathrm{m}^{2}$, wound class, the American Society of Anesthesiologists classification, operative duration, age, and patients with ischemic heart disease, arrhythmia, chronic liver disease, autoimmune disease, blood loss, length of surgery, smoking, and patients that had a preoperative shower [27-29]. Our findings were consistent with known risk factors for SSI previously described (age, duration of surgery, and invasiveness index).

Preoperative antibiotics are proven to be the single most effective measure for preventing SSI in orthopedic and spine surgery [30]. Nonreceipt of preoperative antibiotics is one of the risk factors for SSI, as shown by Olsen et al. [31]. In our study, there was a slight difference between the time of administrating the preoperative antibiotics and wound complication or no complication time (average time: 64 minutes versus 60 minutes, respectively). However, the difference is not statistically different $(p=0.72)$.

Plasmin activity in the circulation distinctly decreased in mice with staphylococcal sepsis [13]. The use of TXA, which is an inhibitor of plasminogen activation, showed aggravation of staphylococcal septic arthritis and sepsis by downregulating fibrinolysis. In humans, it has been found that early inhibition of activated fibrinolysis predicts poor outcomes, including microbial infection, septic shock, and mortality of febrile medical patients [14]. Therefore, the use of anti-fibrinolytic agents in patients with substantial blood loss due to DIC is generally not recommended [32].

In the clinical literature, there is a large block of evidence supporting the safety and effectiveness of intraoperative TXA use in different surgical disciplines. TXA reduces operative bleeding and complications. In spine surgery, TXA reduces blood loss, the amount of transfused blood required, and the rate of blood transfusion without any other increased risk [12]. The future challenge for further investigation is the use of TXA in spine surgeries and revision of patients with sepsis in cases of epidural abscess, vertebral osteomyelitis, or other infected orthopedic surgeries. To our knowledge, this issue has not yet been investigated.

The limitations of this study are the retrospective singlecenter design and the low number of patients. Further prospective multicenter studies should be conducted.

\section{Conclusions}

Despite in vitro studies and evidence in medical patients and animals with sepsis, we have shown that treatment with TXA might exacerbate their clinical status. We found no difference in wound healing and wound complications in spine surgeries with preoperative TXA use.

\section{Conflict of Interest}

No potential conflict of interest relevant to this article was reported.

\section{References}

1. Mehta AI, Babu R, Karikari IO, et al. 2012 Young Investigator Award winner: the distribution of body mass as a significant risk factor for lumbar spinal fusion postoperative infections. Spine (Phila Pa 1976) 2012;37:1652-6.

2. Ousey KJ, Atkinson RA, Williamson JB, Lui S. Negative pressure wound therapy (NPWT) for spinal 
wounds: a systematic review. Spine J 2013;13:1393405.

3. Maruo K, Berven SH. Outcome and treatment of postoperative spine surgical site infections: predictors of treatment success and failure. J Orthop Sci 2014;19:398-404.

4. Lee MJ, Cizik AM, Hamilton D, Chapman JR. Predicting surgical site infection after spine surgery: a validated model using a prospective surgical registry. Spine J 2014;14:2112-7.

5. Prokuski L. Prophylactic antibiotics in orthopaedic surgery. J Am Acad Orthop Surg 2008;16:283-93.

6. Rohrich RJ, Cho MJ. The role of tranexamic acid in plastic surgery: review and technical considerations. Plast Reconstr Surg 2018;141:507-15.

7. Gerstein NS, Brierley JK, Windsor J, et al. Antifibrinolytic agents in cardiac and noncardiac surgery: a comprehensive overview and update. J Cardiothorac Vasc Anesth 2017;31:2183-205.

8. Franchini M, Mengoli C, Marietta M, et al. Safety of intravenous tranexamic acid in patients undergoing majororthopaedic surgery: a meta-analysis of randomised controlled trials. Blood Transfus 2018;16:3643.

9. Jones KE, Butler EK, Barrack T, et al. Tranexamic acid reduced the percent of total blood volume lost during adolescent idiopathic scoliosis surgery. Int J Spine Surg 2017;11:27.

10. Kuo LT, Hsu WH, Chi CC, Yoo JC. Tranexamic acid in total shoulder arthroplasty and reverse shoulder arthroplasty: a systematic review and meta-analysis. BMC Musculoskelet Disord 2018;19:60.

11. Topsoee MF, Settnes A, Ottesen B, Bergholt T. A systematic review and meta-analysis of the effect of prophylactic tranexamic acid treatment in major benign uterine surgery. Int J Gynaecol Obstet 2017;136:1207.

12. Alajmi T, Saeed H, Alfaryan K, Alakeel A, Alfaryan T. Efficacy of tranexamic acid in reducing blood loss and blood transfusion in idiopathic scoliosis: a systematic review and meta-analysis. J Spine Surg 2017;3:531-40.

13. Klak M, Anakkala N, Wang W, et al. Tranexamic acid, an inhibitor of plasminogen activation, aggravates staphylococcal septic arthritis and sepsis. Scand J Infect Dis 2010;42:351-8.

14. Raaphorst J, Johan Groeneveld AB, Bossink AW,
Erik Hack C. Early inhibition of activated fibrinolysis predicts microbial infection, shock and mortality in febrile medical patients. Thromb Haemost 2001;86:543-9.

15. Frossing S, Rono B, Hald A, Romer J, Lund LR. Skin wound healing in MMP2-deficient and MMP2/ plasminogen double-deficient mice. Exp Dermatol 2010;19:e234-40.

16. Green KA, Almholt K, Ploug M, et al. Profibrinolytic effects of metalloproteinases during skin wound healing in the absence of plasminogen. J Invest Dermatol 2008;128:2092-101.

17. Lund LR, Green KA, Stoop AA, et al. Plasminogen activation independent of UPA and tPA maintains wound healing in gene-deficient mice. EMBO J 2006;25:2686-97.

18. Romer J, Bugge TH, Pyke C, et al. Plasminogen and wound healing. Nat Med 1996;2:725.

19. Cao G, Huang Q, Huang Z, et al. The efficacy and safety of multiple-dose oral tranexamic acid on blood loss following total hip arthroplasty: a randomized controlled trial. Int Orthop 2019;43:299-305.

20. Zhu J, Zhu Y, Lei P, Zeng M, Su W, Hu Y. Efficacy and safety of tranexamic acid in total hip replacement: a PRISMA-compliant meta-analysis of 25 randomized controlled trials. Medicine (Baltimore) 2017;96:e9552.

21. Amer KM, Rehman S, Amer K, Haydel C. Efficacy and safety of tranexamic acid in orthopaedic fracture surgery: a meta-analysis and systematic literature review. J Orthop Trauma 2017;31:520-5.

22. Lewis CJ, Li P, Stewart L, et al. Tranexamic acid in life-threatening military injury and the associated risk of infective complications. Br J Surg 2016;103:366-73.

23. Horan TC, Gaynes RP, Martone WJ, Jarvis WR, Emori TG. CDC definitions of nosocomial surgical site infections, 1992: a modification of CDC definitions of surgical wound infections. Infect Control Hosp Epidemiol 1992;13:606-8.

24. Centers for Disease Control and Prevention. National Healthcare Safety Network (NHSN) patient safety component manual. Atlanta (GA): Centers for Disease Control and Prevention; 2020.

25. Mirza SK, Deyo RA, Heagerty PJ, Turner JA, Lee LA, Goodkin R. Towards standardized measurement of adverse events in spine surgery: conceptual model 
and pilot evaluation. BMC Musculoskelet Disord 2006;7:53.

26. Cizik AM, Lee MJ, Martin BI, et al. Using the spine surgical invasiveness index to identify risk of surgical site infection: a multivariate analysis. J Bone Joint Surg Am 2012;94:335-42.

27. Lieber B, Han B, Strom RG, et al. Preoperative predictors of spinal infection within the national surgical quality inpatient database. World Neurosurg 2016;89:517-24.

28. Klemencsics I, Lazary A, Szoverfi Z, Bozsodi A, Eltes P, Varga PP. Risk factors for surgical site infection in elective routine degenerative lumbar surgeries. Spine J 2016;16:1377-83.
29. Wang T, Wang H, Yang DL, Jiang LQ, Zhang LJ, Ding WY. Factors predicting surgical site infection after posterior lumbar surgery: a multicenter retrospective study. Medicine (Baltimore) 2017;96:e6042.

30. Shaffer WO, Baisden JL, Fernand R, Matz PG; North American Spine Society. An evidence-based clinical guideline for antibiotic prophylaxis in spine surgery. Spine J 2013;13:1387-92.

31. Olsen MA, Nepple JJ, Riew KD, et al. Risk factors for surgical site infection following orthopaedic spinal operations. J Bone Joint Surg Am 2008;90:62-9.

32. Mannucci PM, Levi M. Prevention and treatment of major blood loss. N Engl J Med 2007;356:2301-11. 